\title{
On the distribution of some Euler-Mahonian statistics
}

\author{
Alexander Burstein
}

\begin{abstract}
We give a direct combinatorial proof of the equidistribution of two pairs of permutation statistics, (des, aid) and (lec, inv), which have been previously shown to have the same joint distribution as (exc,maj), the major index and the number of excedances of a permutation. Moreover, the triple ( $\mathrm{pix}, \mathrm{lec}, \mathrm{inv}$ ) was shown to have the same distribution as (fix,exc,maj), where fix is the number of fixed points of a permutation. We define a new statistic aix so that our bijection maps (pix, lec, inv) to (aix, des, aid). We also find an Eulerian partner das for a Mahonian statistic mix defined using mesh patterns, so that (das, mix) is equidistributed with (des, inv).

AMS 2000 SUBJECT CLASSIFICATIONS: Primary 05A05; secondary 05A15. KeYwords AND PHRASES: Permutation statistic, Eulerian, Mahonian, admissible inversion, descent, hook factorization, pattern.
\end{abstract}

\section{Introduction}

A combinatorial statistic on a set $S$ is a map $\mathbf{f}: S \rightarrow \mathbb{N}^{m}$ for some integer $m \geq 0$. The distribution of $\mathbf{f}$ is the map $\mathrm{d}_{\mathbf{f}}: \mathbb{N}^{m} \rightarrow \mathbb{N}$ with $\mathrm{d}_{\mathbf{f}}(\mathbf{i})=\left|\mathbf{f}^{-1}(\mathbf{i})\right|$ for $\mathbf{i} \in \mathbb{N}^{m}$, where $\left|\mathbf{f}^{-1}(\mathbf{i})\right|$ is the number of objects $s \in S$ such that $\mathbf{f}(s)=\mathbf{i}$. We say that statistics $\mathbf{f}$ and $\mathbf{g}$ are equidistributed and write $\mathbf{f} \sim \mathbf{g}$ if $\mathrm{d}_{\mathbf{f}}=\mathrm{d}_{\mathbf{g}}$.

Let $\mathfrak{S}_{n}$ be the set of permutations of $[n]=\{1, \ldots, n\}$. The four classic combinatorial statistics on $\mathfrak{S}_{n}$, the number of descents (des), the number of excedances (exc), the number of inversions (inv), and the major index (maj), are defined as follows:

$$
\begin{array}{lll}
\operatorname{Des} \pi=\{i: \pi(i)>\pi(i+1)\}, & \operatorname{des} \pi=|\operatorname{Des} \pi|, \\
\operatorname{Exc} \pi=\{i: \pi(i)>i\}, & \operatorname{exc} \pi=|\operatorname{Exc} \pi|, \\
\operatorname{Inv} \pi=\{(i, j): i<j \text { and } \pi(i)>\pi(j)\}, & \operatorname{inv} \pi=|\operatorname{Inv} \pi|, \\
& \operatorname{maj} \pi=\sum_{i \in \operatorname{Des} \pi} i .
\end{array}
$$

arXiv: 1402.3619 
The set Des $\pi$ is called the descent set of $\pi$, and its elements are called descents. If $i$ is a descent of $\pi$, then $\pi(i)$ and $\pi(i+1)$ are called descent top and descent bottom, respectively. The terminology for the other two sets, $\operatorname{Inv} \pi$ and Exc $\pi$, is similar. When the context is unambiguous, we may refer to the pair $\pi(i) \pi(i+1)$ as a descent or the pair $\pi(i) \pi(j)$ as an inversion.

A statistic with the same distribution as des (such as exc) is called Eulerian, and a statistic with the same distribution as inv (such as maj [8]) is called Mahonian. If eul is Eulerian and mah is Mahonian, then the pair (eul, mah) is called an Euler-Mahonian statistic.

A problem frequently considered since [2] is as follows: given a known Euler-Mahonian statistic $\left(\mathrm{eul}_{1}, \mathrm{mah}_{1}\right)$ and another Eulerian (resp. Mahonian) statistic eul $\mathrm{l}_{2}$ (resp. $\mathrm{mah}_{2}$ ), to find its Mahonian (resp. Eulerian) partner $\operatorname{mah}_{2}$ (resp. eul $)_{2}$ so that $\left(\mathrm{eul}_{1}, \mathrm{mah}_{1}\right) \sim\left(\mathrm{eul}, \mathrm{mah}_{2}\right)$. In this paper, we will give two bijective proofs of equidistribution of two such pairs of bistatistics. In Section 2, we give a direct proof of a bijection between two statistics previously shown to have the same distribution as (exc,maj), and in Section 3 we find an Eulerian partner das for a statistic mix recently defined by Brändén and Claesson [1] using mesh patterns so that (das, mix $) \sim($ des, inv $)$.

\section{Equidistribution of (des, aid) and (lec, inv)}

Of the four pairs $\left(\mathrm{eul}_{1}, \mathrm{mah}_{1}\right)$ involving des or exc and inv or maj, the last to be considered was the pair (exc,maj). First, Shareshian and Wachs [9] found a Mahonian statistic aid such that (exc, maj) (des, aid), and soon afterwards Foata and Han [3] proved that (exc, maj) (lec, inv) for an Eulerian statistic lec defined earlier by Gessel [4] and related to the hook factorization of a permutation. In fact, Foata and Han proved a more refined result that $(\mathrm{fix}, \mathrm{exc}, \mathrm{maj}) \sim(\mathrm{pix}, \mathrm{lec}, \mathrm{inv})$, where $\mathrm{fix} \pi$ is the number of fixed points of $\pi$ and pix $\pi$ is another statistic related to hook factorization of $\pi$.

We will now define the statistics aid, lec and pix.

Definition 2.1. An inversion $(i, j) \in \operatorname{Inv} \pi$ is admissible if either $\pi(j)<$ $\pi(j+1)$ or $\pi(j)>\pi(k)$ for some $i<k<j$. Let Ai $\pi$ be the set of admissible inversions of $\pi$, and let

$$
\text { ai } \pi=|\operatorname{Ai} \pi|, \quad \text { aid } \pi=\text { ai } \pi+\operatorname{des} \pi
$$

Definition 2.2. A string $w=w_{1} w_{2} \ldots w_{r}(r \geq 2)$, over a totally ordered alphabet is a hook if $w_{1}>w_{2} \leq w_{3} \leq \cdots \leq w_{r}$. Every string $\pi$ over $\mathbb{N}$ (and 
hence any permutation $\pi$ ) can be decomposed uniquely [4] as $\pi=\pi_{0} \pi_{1} \ldots \pi_{k}$ $(k \geq 0)$, where $\pi_{0}$ is a (possibly empty) nondecreasing string and each of $\pi_{i}$, $1 \leq i \leq k$, is a hook. Then $\pi_{0} \pi_{1} \ldots \pi_{k}$ is called the hook factorization of $\pi$.

It is easy to see that the hook factorization is unique for any $\pi$, since either $\pi=\pi_{0}$ or we can recursively find the rightmost hook of $\pi$, which starts with the rightmost descent top of $\pi$. The statistics lec and pix are defined as follows:

$$
\operatorname{lec} \pi=\sum_{i=1}^{k} \operatorname{inv} \pi_{i}, \quad \operatorname{pix} \pi=\left|\pi_{0}\right|
$$

where $\left|\pi_{0}\right|$ is the length of $\pi_{0}$.

Shareshian and Wachs [9] gave a proof of (des, aid) (exc, maj) using tools from poset topology such as lexicographic shellability. Subsequently, Foata and Han [3] gave a two-step proof of $(\mathrm{fix}, \mathrm{exc}, \mathrm{maj}) \sim(\mathrm{pix}, \mathrm{lec}, \mathrm{inv})$. The first step of that proof was a bijection on $\mathfrak{S}_{n}$ showing the equidistribution $(\mathrm{fix}, \mathrm{exc}, \mathrm{maj}) \sim(\mathrm{pix}, \mathrm{lec}, \mathrm{imaj})$ (and, in fact, a more refined result that $(\mathrm{fix}$, exc, des, maj $) \sim($ pix, lec, ides, imaj)), where $\operatorname{imaj}(\pi)=$ $\operatorname{maj}\left(\pi^{-1}\right)$ and $i \operatorname{des}(\pi)=\operatorname{des}\left(\pi^{-1}\right)$, using Lyndon words and the word analogs of Kim-Zeng [5] permutation decomposition and hook factorization. The second step was a bijection on $\mathfrak{S}_{n}$ showing that (pix, lec, imaj) (pix, lec, inv).

Somewhat surprisingly, a direct bijective proof of (des, aid) $\sim($ lec, inv) is simpler than any of the bijections mentioned above. We give such a proof and, in fact, find a new statistic aix that is a fix-partner for (des, aid), i.e. such that $($ aix, des, aid $) \sim($ pix, lec, inv $) \sim($ fix, exc, maj $)$.

The statistic aix is defined as follows. Consider the set $\mathbb{N}^{*}$ of all strings in $\mathbb{N}$. Given a string $\pi \in \mathbb{N}^{*}$, let $m$ be the smallest letter in $\pi$ and let $\alpha$ be the maximal left prefix of $\pi$ not containing $m$, so that $\pi=\alpha m \beta$ for some string $\beta$. Then we recursively define aix $\emptyset=0$ and, for $\pi \neq \emptyset$,

$$
\operatorname{aix} \pi= \begin{cases}1+\operatorname{aix} \beta, & \text { if } \alpha=\emptyset \\ \operatorname{aix} \alpha, & \text { if } \alpha \neq \emptyset, \beta \neq \emptyset \\ 0, & \text { if } \alpha \neq \emptyset, \beta=\emptyset\end{cases}
$$

In particular, if $\alpha=\beta=\emptyset$, then $\operatorname{aix} \pi=\operatorname{aix} m=1+\operatorname{aix} \emptyset=1+$ $0=1$. Consider another example: $\operatorname{aix}(2589637 \underline{14})=\operatorname{aix}(2589637)=1+$ $\operatorname{aix}(5896 \underline{3} 7)=1+\operatorname{aix}(\underline{5896})=1+1+\operatorname{aix}(89 \underline{6})=1+1+0=2($ the smallest letters at each step are underlined). 
Proposition 2.3. For any $\pi \in \mathbb{N}^{*}$, we have aix $\pi \leq 1+\operatorname{pix} \pi$.

Proof. The value of aix $\pi$ is at most the length of $\rho$, the maximal nondecreasing left prefix of $\pi$. Since the leftmost hook of $\pi$ starts either at the leftmost descent or at the second-leftmost descent (only if it immediately follows the leftmost descent), it follows that the length of $\rho$ is either pix $\pi$ or $1+\operatorname{pix} \pi$.

We also note that computation of statistics inv, lec, pix, aid, des, aix, involves only comparisons of values of letters or values of positions, but not values of a letter and a position (as in the computation of exc), so that these statistics can be extended to any string of distinct letters.

\subsection{The bijection}

Let $S$ be a set of distinct letters and $k \notin S$ be such that $S \cup\{k\}$ is totally ordered. Let $\tau$ be a permutation of $S$. Let $m$ be the smallest letter in $S \cup\{k\}$. Define the permutation $f(k, \tau)$ of $S \cup\{k\}$ recursively as follows: $f(k, \emptyset)=k$ and

$$
f(k, \tau)= \begin{cases}f(k, \alpha) m \beta, & \text { if } \tau=\alpha m \beta, k>m, \alpha \neq \emptyset, \beta \neq \emptyset \\ f(k, \beta) m, & \text { if } \tau=m \beta, k>m, \\ k m \alpha, & \text { if } \tau=\alpha m, k>m, \\ k \tau, & \text { if } k=m .\end{cases}
$$

Note that both (2.2b) and (2.2c) yield $f(k, m)=k m$ when $k>m$ and $\alpha=\beta=\emptyset$. Now, for $\pi \in \mathfrak{S}_{n}$, define $\phi_{0}(\pi)=\emptyset$ and $\phi_{k}(\pi)=f(\pi(n-k+$ $\left.1), \phi_{k-1}(\pi)\right), k=1, \ldots, n$. Finally, let $\phi(\pi)=\phi_{n}(\pi) \in \mathfrak{S}_{n}$. It is easy to see that for any fixed $k \notin S, f(k, \cdot)$ is a bijection between permutations of $S$ and permutations of $S \cup\{k\}$ starting with $k$. Thus, $\phi$ is also a bijection on $\mathfrak{S}_{n}$.

Let ini $\pi=\pi(1)$. Then we have that

Theorem 2.4. (ini, aix, des, aid) $\phi(\pi)=($ ini, pix, lec, inv) $\pi$.

We will split the proof of the theorem into several parts.

Lemma 2.5. ini $\phi(\pi)=$ ini $\pi$.

Proof. Note that $f(k, \emptyset)=k$, so by the definition of $f$ and induction on the size of $\tau$ we get that $f(k, \tau)$ starts with $k$. Thus, $\phi(\pi)$ starts with $\pi(1)$.

Given a string $\pi$ over a totally ordered alphabet define $k$-suffix of $\pi$, $s_{k}(\pi)$, to be the block of $k$ rightmost letters of $\pi$. Also, define $\pi_{<k}$ (resp. $\pi_{>k}$ ) to be the subsequence of $\pi$ consisting of letters of $\pi$ that are less (resp. greater) than $k$. 
Lemma 2.6. aid $f(k, \tau)=\operatorname{aid} \tau+\left|\tau_{<k}\right|$.

Proof. We will prove this lemma by induction on the length of $\tau$. Clearly, the lemma is true for $\tau=\emptyset$. Assume that the lemma holds for all strings of distinct letters of length less than $|\tau|$. Let $m=\min \tau$ and consider each case in the definition of $f(k, \tau)$.

Case (a). Suppose that $\tau=\alpha m \beta, k>m, \alpha \neq \emptyset, \beta \neq \emptyset$. Then $f(k, \tau)=$ $f(k, \alpha) m \beta$, so by Lemma 2.5, $f(k, \alpha m \beta)=k \hat{\alpha} m \beta$ for some permutation $\hat{\alpha}$ of $\alpha$. By induction (since $|\alpha|<|\tau|$ ), we have

$$
\text { aid } f(k, \alpha)=\operatorname{aid} \alpha+\left|\alpha_{<k}\right| \text {. }
$$

Consider the inversions $a b$ in $\tau$ that are from $\alpha$ to $m \beta$, i.e. those where the inversion top is $a \in \alpha$ and the inversion bottom is $b \in m \beta$ (so $a>b$ ). If $b=$ $m$, then it is followed by an ascent, and hence any inversion with inversion bottom $m$ is admissible (and the number of such (admissible) inversions in $\tau$ is $|\alpha|)$. If $b \in \beta$, then $m<b$ and $m$ is between $a$ and $b$ in $\tau$, so the inversion $a b$ is admissible. Thus, all inversions from $\alpha$ to $m \beta$ are admissible.

Since $\hat{\alpha}$ is a permutation of $\alpha$, we likewise have that all inversions in $f(k, \tau)$ from $\hat{\alpha}$ to $m \beta$ are admissible and, in fact, are the same inversions as the inversions from $\alpha$ to $m \beta$ in $\tau$. Moreover, since $\alpha>m$ (i.e. every letter in $\alpha$ is greater than $m$ ) and $f$ does not change the suffix $m \beta$ of $\tau$, it follows that the number of admissible inversions in $m \beta$ and the number of descents with descent bottoms in $m \beta$ are the same in $\tau$ and $f(k, \tau)$.

Thus, the only remaining pairs left to consider are inversions from $k$ to $m \beta$. As above, we see that all inversions from $k$ to $m \beta$ are admissible, and the number of such inversions is exactly $\left|m \beta_{<k}\right|$. Therefore,

$$
\text { aid } f(k, \tau)-\text { aid } \tau=\left|\alpha_{<k}\right|+\left|m \beta_{<k}\right|=\left|\alpha_{<k} m \beta_{<k}\right|=\left|\tau_{<k}\right|,
$$

as desired.

Case (b). Suppose that $\tau=m \beta$ and $k>m$. Then $f(k, \tau)=f(k, \beta) m=$ $k \hat{\beta} m$ for some permutation $\hat{\beta}$ of $\beta$. As before, we have by induction that

$$
\text { aid } f(k, \beta)=\operatorname{aid} \beta+\left|\beta_{<k}\right| \text {. }
$$

Since $k \hat{\beta}>m$ and $m$ is last in $k \hat{\beta} m$, it follows that no admissible inversion ends on $m$. Thus, ai $f(k, \beta) m=$ ai $f(k, \beta)$ and des $f(k, \beta) m=\operatorname{des} f(k, \beta)+$ 1 , where 1 counts the last descent to $m$. Finally, aid $\tau=$ aid $m \beta=$ aid $\beta$ since $m<\beta$ and hence no inversion (or descent) of $\tau$ begins with $m$. Therefore, 


$$
\begin{aligned}
\text { aid } f(k, \tau)=\text { aid } f(k, \beta)+1 & =\operatorname{aid} \beta+\left|\beta_{<k}\right|+1 \\
& =\operatorname{aid} m \beta+\left|m \beta_{<k}\right|=\operatorname{aid} \tau+\left|\tau_{<k}\right| .
\end{aligned}
$$

Case (c). Suppose that $\tau=\alpha m, k>m$. Then $f(k, \tau)=k m \alpha$. Thus, the descents of $f(k, \tau)$ are obtained from descents of $\tau$ by replacing the descent from the right letter of $\alpha$ to $m$ with the descent $k m$, so des $f(k, \tau)=\operatorname{des} \tau$. As in Case (b), no admissible inversion of $\tau$ ends on $m$, and, as in Cases (a) and (b), all inversions from $k$ to $m \alpha$ are admissible. Thus,

$$
\text { ai } f(k, \tau)=\text { ai } k m \alpha=\text { ai } m \alpha+\left|m \alpha_{<k}\right|=\text { ai } \alpha m+\left|\alpha_{<k} m\right|=\text { ai } \tau+\left|\tau_{<k}\right|,
$$

so

aid $f(k, \tau)=$ ai $f(k, \tau)+\operatorname{des} f(k, \tau)=$ ai $\tau+\left|\tau_{<k}\right|+\operatorname{des} \tau=\operatorname{aid} \tau+\left|\tau_{<k}\right|$.

Case (d). If $k<\tau$, then no inversion (or descent) of $f(k, \tau)=k \tau$ starts with $k$, and $\left|\tau_{<k}\right|=0$, so aid $f(k, \tau)=$ aid $\tau=\operatorname{aid} \tau+\left|\tau_{<k}\right|$. This ends the proof.

Lemma 2.7. aid $\phi(\pi)=\operatorname{inv} \pi$.

Proof. Applying Lemma 2.6 repeatedly, we obtain

$$
\operatorname{aid} \phi(\pi)=\sum_{k=0}^{n-1}\left|\phi_{k}(\pi)_{<\pi(n-k)}\right| \text {. }
$$

But each $\phi_{k}(\pi)$ is a permutation of $s_{k}(\pi)$, so

$$
\operatorname{aid} \phi(\pi)=\sum_{k=0}^{n-1}\left|s_{k}(\pi)_{<\pi(n-k)}\right| .
$$

Each summand on the right is the number of inversions of $\pi$ with inversion top $\pi(n-k)$. Summing over $k=0,1 \ldots, n-1$, we get aid $\phi(\pi)=\operatorname{inv}(\pi)$, as desired.

For a string $\sigma$ and a letter $l$, write $\sigma>l$ if every letter in $\sigma$ is greater than $l$. Consider the descents of $\tau$ and $f(k, \tau)$ in each case of the definition of $f$. In case $(2.2 \mathrm{a})$, we have $\alpha>m$ and $f(k, \tau)=f(k, \alpha m \beta)=f(k, \alpha) m \beta$, so the descent bottoms in the right prefix $m \beta$ of both $\tau$ and $f(k, \tau)$ are the same, and hence

$$
\operatorname{des} f(k, \tau)-\operatorname{des} \tau=\operatorname{des} f(k, \alpha)-\operatorname{des} \alpha .
$$

Note that in this case $\operatorname{aix} \tau=\operatorname{aix} \alpha$ and $\operatorname{aix} f(k, \tau)=\operatorname{aix} f(k, \alpha)$. 
In case $(2.2 \mathrm{~b})$, des $\tau=\operatorname{des} m \beta=\operatorname{des} \beta$ since $\beta=\emptyset$ or $m<\beta$. However, $\operatorname{des} f(k, \tau)=\operatorname{des} f(k, \beta) m=\operatorname{des} f(k, \beta)+1$ since $f(k, \beta)=k \hat{\beta}$ for some permutation $\hat{\beta}$ of $\beta$ and hence $f(k, \beta)>m$. Thus,

$$
\operatorname{des} f(k, \tau)-\operatorname{des} \tau=\operatorname{des} f(k, \beta)-\operatorname{des} \beta+1 .
$$

Note that in this case aix $f(k, \tau)=0$, and $\operatorname{aix} \tau=1+\operatorname{aix} \beta>0$.

In case $(2.2 \mathrm{c})$, let $a$ be the last letter of $\alpha$. Then the descents of $f(k, \tau)=$ $k m \alpha, \alpha \neq \emptyset$ are obtained from the descents of $\tau=\alpha m$ by replacing the descent am with the descent $k m$. Thus, des $f(k, \tau)=\operatorname{des} \tau=\operatorname{des} \alpha+1$, and hence

$$
\operatorname{des} f(k, \tau)-\operatorname{des} \tau=0 .
$$

Note that in this case aix $\tau=0$ and aix $f(k, \tau)=\operatorname{aix} k=1=1+\operatorname{aix} \tau$.

In case $(2.2 \mathrm{~d}), f(k, \tau)=k \tau$, and $k<\tau$, so des $f(k, \tau)=\operatorname{des} \tau$, and hence again

$$
\operatorname{des} f(k, \tau)-\operatorname{des} \tau=0 .
$$

Note that in this case aix $f(k, \tau)=\operatorname{aix} \tau+1>0$.

Finally, des $f(k, \emptyset)-\operatorname{des} \emptyset=0-0=0$. Thus, we can see by induction on the length of $\tau$ that

$$
\operatorname{des} f(k, \tau)-\operatorname{des} \tau \geq 0
$$

for any string $\tau$ of distinct letters, and the difference stays the same or increases by 1 with each application of rules $(2.2 \mathrm{a})$ or $(2.2 \mathrm{~b})$, respectively.

Lemma 2.8. We have des $f(k, \tau)=\operatorname{des} \tau$ if and only if $\operatorname{aix} f(k, \tau)=$ $\operatorname{aix} \tau+1>0$, and des $f(k, \tau)>\operatorname{des} \tau$ if and only if $\operatorname{aix} f(k, \tau)=0$.

Proof. Case 1. Suppose that des $f(k, \tau)=\operatorname{des} \tau$. Then it follows from the above argument that the computation of $f(k, \tau)$ involves no application of $(2.2 \mathrm{~b})$, i.e. a repeated application of $(2.2 \mathrm{a})$ (possibly zero times) followed by a single application of $(2.2 \mathrm{c})$ or $(2.2 \mathrm{~d})$ or $f(k, \emptyset)=k$. The conditions in the case $(2.2 \mathrm{a})$ are the same as in the case $(2.1 \mathrm{~b})$, so applying $(2.2 \mathrm{a})$ repeatedly, we obtain either

- a prefix $\alpha^{\prime} m^{\prime}$ of $\tau$ such that $\alpha \neq \emptyset, \alpha^{\prime}>m^{\prime}, k>m^{\prime}$, aix $\tau=\operatorname{aix} \alpha^{\prime} m^{\prime}$ and aix $f(k, \tau)=\operatorname{aix} f\left(k, \alpha^{\prime} m^{\prime}\right)$, or

- a prefix $\alpha^{\prime \prime}$ of $\tau$ such that $k<\alpha^{\prime \prime}, \operatorname{aix} \tau=\operatorname{aix} \alpha^{\prime \prime}$ and $\operatorname{aix} f(k, \tau)=$ $\operatorname{aix} f\left(k, \alpha^{\prime \prime}\right)$.

In the former case, we have aix $\tau=\operatorname{aix} \alpha^{\prime} m^{\prime}=0$ and aix $f(k, \tau)=$ $\operatorname{aix} f\left(k, \alpha^{\prime} m^{\prime}\right)=\operatorname{aix} k m^{\prime} \alpha^{\prime}=\operatorname{aix} k=1=1+\operatorname{aix} \tau$. In the latter case, 
we have $\operatorname{aix} f\left(k, \alpha^{\prime \prime}\right)=\operatorname{aix} k \alpha^{\prime \prime}=1+\operatorname{aix} \alpha^{\prime \prime}=1+\operatorname{aix} \tau$. Thus, in either case, $\operatorname{des} f(k, \tau)=\operatorname{des} \tau$ implies aix $f(k, \tau)=\operatorname{aix} \tau+1$. The converse is proved similarly.

Case 2. Suppose that $\operatorname{des} f(k, \tau)>\operatorname{des} \tau$. Then the computation of $f(k, \tau)$ starts with a repeated application of $(2.2 \mathrm{a})$ (possibly zero times) followed by an application of (2.2b) (after which the process may still continue). Thus, as before, after repeated application of $(2.2 \mathrm{a})$, we obtain a prefix $m^{\prime} \beta^{\prime}$ of $\tau$ such that $k>m^{\prime}, m^{\prime}<\beta^{\prime}$ and aix $f(k, \tau)=$ aix $f\left(k, m^{\prime} \beta^{\prime}\right)=\operatorname{aix} f\left(k, \beta^{\prime}\right) m^{\prime}$. But $f\left(k, \beta^{\prime}\right)=k \hat{\beta}^{\prime}$ for some permutation $\hat{\beta}^{\prime}$ of $\beta^{\prime}$, so $f\left(k, \beta^{\prime}\right)>m^{\prime}$, and hence aix $f\left(k, \beta^{\prime}\right) m^{\prime}=0$, which in turn implies that aix $f(k, \tau)=0$, as desired. The converse is proved similarly.

Lemma 2.9. If $\operatorname{aix} \tau=0$, then for any $k$, we have aix $f(k, \tau)=1$ and $\operatorname{des} f(k, \tau)=\operatorname{des} \tau$.

Proof. The lemma is obviously true for $\tau=\emptyset$. Suppose $\tau \neq \emptyset$. Since aix $\tau=$ 0 , it follows that $\tau=\alpha m_{0} m_{1} \beta_{1} \ldots m_{r} \beta_{r}$, where $\alpha \neq \emptyset, \alpha>m_{0}$, and if $r \geq 1$, then $\beta_{i} \neq \emptyset$ and $m_{i}<\beta_{i}$ for all $i=1, \ldots, r$, and $m_{0}>m_{1}>\cdots>m_{r}$. If $k>m_{0}$, then applying $(2.2 \mathrm{a})$ repeatedly, followed by $(2.2 \mathrm{c})$, we obtain

$$
\begin{aligned}
f(k, \tau)=f\left(k, \alpha m_{0} m_{1} \beta_{1} \ldots m_{r} \beta_{r}\right) & \\
& =f\left(k, \alpha m_{0}\right) m_{1} \beta_{1} \ldots m_{r} \beta_{r}=k m_{0} \alpha m_{1} \beta_{1} \ldots m_{r} \beta_{r}
\end{aligned}
$$

so that aix $f(k, \tau)=\operatorname{aix} k m_{0} \alpha=\operatorname{aix} k=1$. Also, all descent bottoms of $\tau$ and $f(k, \tau)$ are the same (including $\left.m_{0}\right)$, so des $f(k, \tau)=\operatorname{des} \tau$.

Suppose that $k<m_{0}$, and let $j$ be maximal such that $k<m_{j}$. Then $k<\alpha m_{0} m_{1} \beta_{1} \ldots m_{j} \beta_{j}$, so

$$
\begin{aligned}
f(k, \tau) & =f\left(k, \alpha m_{0} m_{1} \beta_{1} \ldots m_{r} \beta_{r}\right) \\
& =f\left(k, \alpha m_{0} m_{1} \beta_{1} \ldots m_{j} \beta_{j}\right) m_{j+1} \beta_{j+1} \ldots m_{r} \beta_{r} \\
& =k \alpha m_{0} m_{1} \beta_{1} \ldots m_{j} \beta_{j} m_{j+1} \beta_{j+1} \ldots m_{r} \beta_{r} \\
& =k \tau .
\end{aligned}
$$

Therefore, $f(k, \tau)=k \tau$ starts with an ascent, so des $f(k, \tau)=\operatorname{des} k \tau=$ $\operatorname{des} \tau$ and hence aix $f(k, \tau)=1+\operatorname{aix} \tau=1$ by Lemma 2.8.

Lemma 2.10. Suppose that aix $f(k, \tau)=0$ and $\tau=f(l, \sigma)$ for some letter $l$ and string $\sigma$. Then $\operatorname{des} f(k, \tau)=1+\operatorname{des} f(k, \sigma)$.

Proof. By Lemma 2.9, note that aix $\tau \geq 1$, since otherwise aix $f(k, \tau)=1$. In particular, $\tau \neq \emptyset$, so there is indeed a letter $l$ and a string $\sigma$ such that $\tau=f(l, \sigma)$. 
Since $\tau=f(l, \sigma)$, it follows that $\tau$ starts with $l$. Let $l=m_{0}>m_{1}>$ $\cdots>m_{r}$ be the values of $\tau$ at positions of the left-to-right minima of $\tau$ (i.e. at positions $i$ such that $\tau(j)>\tau(i)$ for $j<i)$. Then $\tau=m_{0} \tau_{0} m_{1} \tau_{1} \ldots m_{r} \tau_{r}$ with $\tau_{i}>m_{i}$ for all $i=0,1, \ldots, r$. We also have that $\tau_{i} \neq \emptyset$ for $i \geq 1$ since otherwise aix $\tau=0$. Therefore,

$$
f(k, \tau)=f\left(k, m_{0} \tau_{0}\right) m_{1} \tau_{1} \ldots m_{r} \tau_{r}=f\left(k, l \tau_{0}\right) m_{1} \tau_{1} \ldots m_{r} \tau_{r}
$$

so $\operatorname{aix} f(k, \tau)=\operatorname{aix} f\left(k, l \tau_{0}\right)$. If $k<l$, then $k<l \tau_{0}$, so $f\left(k, l \tau_{0}\right)=k l \tau_{0}$ and aix $f\left(k, l \tau_{0}\right)=1+\operatorname{aix} l \tau_{0}>0$, which contradicts our assumption. Therefore, $k>l$.

Since aix $f(l, \sigma)=\operatorname{aix} \tau>0$, it follows that the recursive computation of $f(l, \sigma)$ involves no application of $(2.2 \mathrm{~b})$. Thus, we have two cases:

- $\sigma=\alpha l_{1} \beta_{1} \ldots l_{s} \beta_{s}$, where $l>l_{1}>\cdots>l_{s}, \alpha \neq \emptyset, \alpha>l, \beta_{i} \neq \emptyset$ and $\beta_{i}>l_{i}$ for $i=1, \ldots, s$.

- $\sigma=\alpha l_{0} l_{1} \beta_{1} \ldots l_{s} \beta_{s}$, where $l>l_{0}>l_{1}>\cdots>l_{s}, \alpha \neq \emptyset, \alpha>l, \beta_{i} \neq \emptyset$ and $\beta_{i}>l_{i}$ for $i=1, \ldots, s$.

Let $\beta=l_{1} \beta_{1} \ldots l_{s} \beta_{s}$. In the first case, we have

$$
\begin{aligned}
\tau=f(l, \sigma) & =f(l, \alpha \beta)=f(l, \alpha) \beta=l \alpha \beta=l \sigma \\
f(k, \tau) & =f(k, l \alpha \beta)=f(k, l \alpha) \beta=f(k, \alpha) l \beta \\
f(k, \sigma) & =f(k, \alpha \beta)=f(k, \alpha) \beta .
\end{aligned}
$$

Note that ini $\beta=l_{1}<l$. Also note that $f(k, \alpha) l \beta=k \hat{\alpha} l \beta$ for some permutation $\hat{\alpha}$ of $\alpha$. Since $\alpha>l$, it follows that $\hat{\alpha}>l$. Let $a$ be the last letter of $f(k, \alpha)$. Then the descents of $f(k, \alpha) l \beta$ are obtained from the descents of $f(k, \alpha) \beta$ by replacing the descent $a l_{1}$ with the descents $a l$ and $l l_{1}$. Therefore, we have des $f(k, \tau)=\operatorname{des} f(k, \sigma)+1$ as desired.

In the second case, we have

$$
\begin{aligned}
\tau=f(l, \sigma) & =f\left(l, \alpha l_{0} \beta\right)=f\left(l, \alpha l_{0}\right) \beta=l l_{0} \alpha \beta \\
f(k, \tau) & =f\left(k, l l_{0} \alpha \beta\right)=f\left(k, l l_{0} \alpha\right) \beta=f(k, l) l_{0} \alpha \beta=k l l_{0} \alpha \beta \\
f(k, \sigma) & =f\left(k, \alpha l_{0} \beta\right)=f\left(k, \alpha l_{0}\right) \beta=k l_{0} \alpha \beta .
\end{aligned}
$$

Since $k>l>l_{0}$, it is easy to see that $\operatorname{des} f(k, \tau)=\operatorname{des} f(k, \sigma)+1$. This ends the proof.

Lemma 2.11. (aix, des) $\phi(\pi)=($ pix, lec $) \pi$. 
Proof. The proof is by induction on the length of $\pi$. The result is obviously true for $\pi=\emptyset$. Define $g(k, \tau)=k \tau$ for a string $\tau$ of distinct elements and an element $k$ not in the alphabet of $\tau$. Then it is easy to see that the results of Lemmas 2.8, 2.9 and 2.10 hold if we replace $f$ with $g$, aix with pix, and des with lec. This implies the lemma and thus finishes the proof of Theorem 2.4.

Remark 2.12. We note that a statistic rix similar to aix (up to an easy transformation) has been independently defined by Z. Lin [7].

It would be interesting to construct a direct bijection on permutations that maps (aix, des, aid) to (fix, exc, maj).

Remark 2.13. Rawlings major index $r$ maj is a Mahonian statistic that interpolates between maj and inv, and is defined as follows:

$$
\begin{aligned}
& \operatorname{Des}_{r}(\pi)=\{i \in \operatorname{Des}(\pi): \pi(i)-\pi(i+1) \geq r\} \\
& \operatorname{Inv}_{r}(\pi)=\{(i, j) \in \operatorname{Inv}(\pi): \pi(i)-\pi(j)<r\} \\
& \operatorname{rmaj}(\pi)=\sum_{i \in \operatorname{Des}_{r}(\pi)} i+\left|\operatorname{Inv}_{r}(\pi)\right|
\end{aligned}
$$

Note that on $\mathfrak{S}_{n}, 1 \mathrm{maj}=\operatorname{maj}, n$ maj $=\operatorname{inv}$, and $\left|\operatorname{Inv}_{2}(\pi)\right|=\operatorname{ides}(\pi)=$ $\operatorname{des}\left(\pi^{-1}\right)$. It is known [10] that (ides, $\left.2 \mathrm{maj}\right) \sim($ exc, maj $)$. It would be interesting to find a fix-partner $2 \mathrm{fix}$ for (ides, $2 \mathrm{maj}$ ) so that (fix, exc, maj) $(2 \mathrm{fix}$, ides, $2 \mathrm{maj})$. Continuing in the same vein, for $3 \leq r \leq n-1$, it would be interesting to find the interpolating statistics $r \mathrm{fix}$ and $r$ exc so that $(\mathrm{fix}, \mathrm{exc}, \operatorname{maj}) \sim($ fix, $r$ exc,$r \operatorname{maj}) \sim($ pix, lec, inv $)$.

\section{Equidistribution of (das, mix) and (des, inv)}

A Mahonian statistic mix counting some inversions and some noninversions has been defined by P. Brändén, A. Claesson [1]. Even though it was originally defined using mesh patterns, it may be easily defined without using those. Define a left-to-right maximum of $\pi$ to be a position $i$ of $\pi$ such that $\pi(j)<\pi(i)$ for $j<i$. The statistic mix counts pairs defined on a permutation $\pi$ as follows:

- inversions $\pi(i) \pi(j)$ such that $i$ is a left-to-right maximum of $\pi$, and

- non-inversions $\pi(i) \pi(j)$ such that there is a left-to-right-maximum $k<$ $i$ with $\pi(k)>\pi(j)$.

Our definition of mix is the reversal of the mix as originally defined in [1]. However, we think that our definition is preferable, since for the 
identity permutation $\operatorname{id}_{n}=12 \ldots n$, we have $\operatorname{mix}\left(\mathrm{id}_{n}\right)=0$, rather than $\operatorname{mix}\left(\operatorname{id}_{n}\right)=n-1$ under the original definition.

There is also a direct bijection given in [1] that takes inv to mix. Making the necessary minor changes to account for the difference in definitions mentioned above, we describe it as follows.

Let $M=\left\{m_{1}<\cdots<m_{k}\right\}$ be the set of values of left-to-right maxima of $\pi$, and let $B_{i}$ be the set of entries of $\pi$ that are smaller than and to the right of $m_{i}$. Also, for $S \subseteq[n]$, let $\psi_{S}(\pi)$ be the result of reversing the subword of $\pi$ that is a permutation on $S$. Then define

$$
\psi=\psi_{B_{1}} \circ \psi_{B_{2} \cap B_{1}} \circ \cdots \circ \psi_{B_{k-1}} \circ \psi_{B_{k} \cap B_{k-1}} \circ \psi_{B_{k}} .
$$

Then we have [1] that $\psi$ is an involution and $\operatorname{mix} \psi(\pi)=\operatorname{inv} \pi$ (and vice versa).

We observe that there is a natural Eulerian partner das (a mix of descents and ascents) for mix such that (das, mix) $\sim($ des, inv). Let das $\pi$ be the number of positions $i \in[n-1]$ of $\pi$ such that

- $\pi(i) \pi(i+1)$ is a descent, and $i$ is a left-to-right maximum of $\pi$, or

- $\pi(i) \pi(i+1)$ is an ascent, and there is a left-to-right-maximum $k<i$ with $\pi(k)>\pi(i+1)$.

Theorem 3.1. (das, mix) $\psi(\pi)=($ des, inv $) \pi$.

Proof. The proof is easily constructed by induction on $k$, following along the lines of the proof of Theorem 10 in [1]. In fact, our extension of that proof is so routine that we leave it as an exercise for the reader.

Remark 3.2. We also note that a restriction of the map $\psi$ yields Krattenthaler's bijection [6] between 321-avoiding and 312-avoiding permutations on $\mathfrak{S}_{n}$ using Dyck paths (modified up to the suitable reversal and complementation symmetries).

\section{Acknowledgements}

The author would like to thank the anonymous referees for their careful reading of the article as well as helpful comments and suggestions.

\section{References}

[1] P. Brändén, A. Claesson, Mesh patterns and the expansion of permutation statistics as sums of permutation patterns, Electron. J. Combin. 18(2) (2011-2012), \#P5. MR2795782 
[2] D. Foata, Distributions eulériennes et mahoniennes sur le groupe des permutations, in Higher Combinatorics, M. Aigner, ed., vol. 19, D. Reidel Publishing Co., Dordrecht-Holland, 1977, 27-49. MR0519777

[3] D. Foata, G. Han, Fix-Mahonian Calculus, III: a quadruple distribution, Monatshefte für Mathematik 154 (2008), 177-197. MR2413301

[4] I. Gessel, A coloring problem, Amer. Math. Monthly 98 (1991), 530533. MR1109577

[5] D. Kim, J. Zeng, A new decomposition of derangements, J. Combin. Theory Ser. A 96 (2001), 192-198. MR1855792

[6] C. Krattenthaler, Permutations with restricted patterns and Dyck paths, Adv. Appl. Math. 27 (2001), 510-530. MR1868978

[7] Z. Lin, On some generalized $q$-Eulerian polynomials, Electron. J. Combin. 20(1) (2013), \#P55. MR3040617

[8] P. A. MacMahon, Combinatory Analysis, 2 volumes, Cambridge University Press, London, 1915-1916. Reprinted by Chelsea, New York, 1960. MR0141605

[9] J. Shareshian, M. Wachs, q-Eulerian polynomials: excedance number and major index, Electron. Res. Announc. Amer. Math. Soc. 13 (2007), 33-45. MR2300004

[10] M. Wachs, Personal communication.

Alexander Burstein

Department of Mathematics

HOWARD UNIVERSITY

WASHINGTON, DC 20059

USA

E-mail address: aburstein@howard.edu

ReCEIVEd 15 February 2014 